\title{
„Der Querschnitt“ oder: Die Kunst des Sporttreibens
}

\author{
KAI MARCEL SICKS
}

Am Anfang steht ein Kunsthändler: Der Düsseldorfer Galerist Alfred Flechtheim veröffentlicht 1921 fünf Hefte unter dem Titel „Der Querschnitt", mit denen er Freunde und Interessenten über aktuelle Tendenzen der Kunstszene informieren und die bei ihm zum Verkauf gebotenen Werke bewerben will. Die „Marginalien der Galerie Flechtheim“ - so der Untertitel des ersten Heftes - vereinigen Auszüge aus der kunstkritischen Fachpresse und den aktuellen Feuilletons sowie Abbildungen von Gemälden und Skulpturen aus dem Besitz Flechtheims. Der Titel erweist sich als Programm: Das Heft inszeniert einen querschnittartigen Überblick über die zeitgenössische Kunst und die aktuelle Kunsthandel- und Galerieszene.

Schon im Laufe des ersten Jahres überträgt Flechtheim die Herausgeberschaft des „Querschnitts“ an den Kunstkritiker und Romancier Hermann von Wedderkop, unter dessen Ägide die Zeitschrift zu einer zentralen Plattform der deutschen Avantgardebewegung aufsteigt. In seinen Glanzzeiten um 1925 erreicht der „Querschnitt“" eine Auflage von 20.000 Exemplaren und ist damit den bestverkauften Kulturzeitschriften der Weimarer Republik, der „Weltbühne“ und der „Neuen Rundschau“, ebenbürtig. Der Niedergang des „Querschnitts“ beginnt mit der Machtübernahme der Nationalsozialisten, die die Zeitschrift aufgrund ihrer kosmopolitischen und linksbürgerlich-demokratischen Ausrichtung unter Auflagen stellen. Nach erheblichen Umstrukturierungen im Jahr 1933 muss die Produktion 1936 endgültig aufgegeben werden; Wedder- 
kop hat das sinkende Schiff zu diesem Zeitpunkt bereits verlassen (vgl. Deininger/Felder 1998: 30-33).

Sind die ersten „Querschnitt“-Hefte noch ausschließlich mit Texten und Bildern zu Malerei, Skulptur und Galeriewesen gefüllt, so beginnt Ende 1921 eine thematische Ausweitung auf aktuelle Trends der modernen Alltagswelten. Insbesondere der sich soeben als Massenphänomen etablierende Leistungs- und Wettkampfsport rückt nun als zweiter Schwerpunkt neben die Kunst ins Zentrum der Zeitschrift. ${ }^{1}$ Die sich so vollziehende Transgression der Grenze von Eliten- und Massenkultur lässt sich dabei als Effekt eines Programms zur Erneuerung der Kunst verstehen, das die Herausgeber mitunter in programmatischen Beiträgen ausführen, vor allem aber durch die Auswahl und Anordnung der Zeitschriftenbeiträge kommunizieren.

Das Verhältnis von Kunst und Sport im „Querschnitt“ steht im Mittelpunkt der folgenden Ausführungen. Diese zeigen zunächst verschiedene Verfahren auf, durch die der „Querschnitt“ die beiden Kulturpraktiken zusammenführt. Hier schon deutet sich an, dass die Herausgeber der Zeitschrift Sport und Kunst systematisch analogisieren, bisweilen sogar kontaminieren. In einem zweiten Abschnitt soll dieser Spur gefolgt und die Frage nach dem ästhetischen Konzept gestellt werden, das sich hinter der Sport-Kunst-Analogisierung verbirgt. Es zeigt sich, dass die Herausgeber in ihrem vom Sport inspirierten Kunstideal Kategorien wie „Spannung“, „Authentizität“, „Präzision“ und „Amerikanität" in den Vordergrund stellen. Das solchermaßen bestimmte Konzept wird abschließend in seinem diskursiven Kontext, der Diskussion um die Kunst und das Lebensgefühl der „Neuen Sachlichkeit“, situiert. Die Analyse der Sportdarstellung im „Querschnitt“ öffnet so nicht nur den Blick auf das der Zeitschrift zugrunde liegende Gestaltungskonzept, sondern vermag obendrein wesentliche Ursachen der intellektuellen Sportbegeisterung in den zwanziger Jahren zu erhellen.

\section{"Dichter und Sportsmen" - Sport-Kunst- Konstellationen im "Querschnitt"}

Bereits frühe programmatische Bemerkungen propagieren die Präsenz des Sports im „Querschnitt“. So heißt es in einer Fußnote, die die Herausgeber Ende 1921 einem Artikel mit dem Titel „Wie gewinnt der

1 Die hartnäckig kursierende Behauptung, der „Querschnitt“ habe zwischenzeitlich den Untertitel „Magazin für Kunst, Literatur und Boxsport“ geführt (Berg 1997: 22; Berg 1998: 137), lässt sich dagegen nicht belegen. Allenfalls handelt es sich hier um ein zeitgenössisches Rezeptionsurteil. 
Boxsport das Allgemein-Interesse?“ beifügen: „Der Querschnitt hält es für seine Pflicht, den Boxsport auch in den deutschen Künstlerkreisen populär zu machen. In Paris sind Braque, Derain, Dufy, Matisse, Picasso, de Vlaminck begeisterte Anhänger, und Rodin fehlte in kaum einem Kampf" (Der Querschnitt 6/1921: 221). Die Konzentration auf das Boxen, so ausgeprägt sie in den ersten Jahrgängen des Heftes auch erscheint (vgl. Berg 1997: 22; Berg 1998: 137f.), wird dabei allmählich von der Berücksichtigung sämtlicher Disziplinen des Leistungs- und Wettkampfsports abgelöst. ${ }^{2}$ Schon 1922 erklärt Flechtheim in diesem allgemeineren Sinn - und wieder im Rahmen einer syntaktischen Annäherung von Kunst und Sport: „Inständige Bitten verständiger Leser des ,Querschnitts` veranlassen uns, denselben weiter herauszugeben [...]. Er wird Aufsätze über Kunst bringen, über Tanzen und Sport und so weiter" (Der Querschnitt 1/1922: 13).

Aber der „Querschnitt“ enthält nicht nur „Aufsätze“ - die Parallelisierung von Sport und Kunst inszeniert die Zeitschrift vielmehr im Rahmen einer ästhetischen Struktur, die durch ein intrikates Verhältnis von Text und Bild geprägt ist. Bilder finden sich einerseits in Form von Zeichnungen und Lithographien als Einlassungen im Fließtext und andererseits in Form von Fotografien und Gemäldereproduktionen in separaten, jeweils vierseitigen Bildblöcken - wobei jeweils zwei Bilder eine Bildseite füllen. Auch die Texte werden in zwei unterschiedlichen Modulen präsentiert: Der erste, in der Regel nicht betitelte Teil enthält überwiegend feuilletonistische Reportagen, seltener Lyrik und Lieder. Der mit „Marginalien“ überschriebene zweite Teil bietet skurrile Zeitungszitate, Sport-, Theater-, Ausstellungs- und Konzertkritiken (man beachte die Zusammenstellung!), Berichte über gesellschaftliche Ereignisse, Bonmots und Anekdoten. Die meisten der mit Sport befassten Artikel im „Querschnitt“ haben publizistischen Charakter: Sie beschäftigen sich mit dem Regelwerk oder dem öffentlichen Ansehen einzelner Sportarten und setzen sich mit konkreten Sportereignissen und -wettkämpfen auseinander. Die meisten Aufsätze widmen sich einzelnen Sportlern und ihren Karrieren; nicht selten sind diese Beiträge als Erfahrungsberichte von betroffenen Sportlern selbst verfasst.

In jedem Fall bildet die gelegentlich aufscheinende Poetisierung des Sports - in Gedichten, Aphorismen und fiktionalen Prosastücken - ein erstes Verfahren seiner Verknüpfung mit künstlerischen - hier mit litera-

2 Drei Ausgaben des „Querschnitts“ konzentrieren sich dabei ausschließlich auf den Sport: Heft 5/1926 anlässlich der Düsseldorfer Ausstellung für Gesundheitspflege, Soziale Fürsorge und Leibesübungen („Gesolei“), Heft 8/1928 anlässlich der Olympischen Winterspiele in St. Moritz und Heft 6/1932 anlässlich der Olympischen Sommerspiele in Los Angeles. 
rischen - Ausdrucksformen. ${ }^{3}$ Dabei sind etwa die Sportgedichte von einer ironischen Distanz und dem Spiel mit einfachen Metren und Reimschemata geprägt, wie im folgenden, hier deutlich gekürzt wiedergegebenen Gedicht W. Kramers:

„Läufer, Springer, Sprinter, Segler, Handballmänner, Autler, Kegler, Leichtathleten, Turner, Schützen, Boxer, Radler, Hockeyfritzen, [...] Knochenbrüche, schiefe Nasen, Gehen, Laufen, Rennen, Rasen, Stoß und Schwinger, Grade, Haken, Kopf und Schienenbein und Magen. [...] Stemmen, Stoßen, Drücken, Heben, Mitte, Oben, Unten, Neben, Hipp-Hurra, Knock-out und Feste, Mittel, Gute, Besser, Beste, Fünfzig, Hundert, Hunderttausend, Langsam, Schneller, Schnellst und Sausend, Durchschnitt, Leistung und Rekord, Das ist Sport!!“(Der Querschnitt 8/1928: 595).

Die Übersetzung der Steigerungslogik und Monotonie des Sports in die lyrische Struktur führt in diesem Gedicht dazu, dass die Poetisierung des Sports mit einer „Versportlichung“ der Poesie einhergeht. Dass der Sport solchermaßen zum Maßstab für die Kunst wird, ist im „Querschnitt" kein Einzelfall; die späteren Ausführungen werden dies detaillierter erläutern.

Als Motiv findet sich Sport nicht nur in literarischen Texten, sondern auch in zahlreichen im „Querschnitt“ reproduzierten Arbeiten der bildenden Kunst, in denen Sportler/innen neuartige Modelle für Porträts und Studien idealer Körper abgeben. Die vertretenen Künstler entstammen häufig dem engeren Kreis um Flechtheim in Berlin, wie etwa die Bildhauerin Renée Sintenis („Der Boxer Erich Brandl“, Statuette, Der Querschnitt 2/1926: o.P.) oder die Maler Rudolf Grossmann („Schattenboxer“, Lithographie, Der Querschnitt 6/1921: 219) und George Grosz („Boxer“, Ölgemälde, Der Querschnitt 5/1926: o.P.). Fast ebenso oft

3 Aus dem Roman „Spiel um Liebe“ der französischen Star-Tennisspielerin Suzanne Lenglen geben die Herausgeber des „Querschnitts“ eine „bezeichnende Stelle" wieder (Der Querschnitt 5/1928: 338), in der die heftig umworbene Protagonistin und Tennisspielerin Marcelle einen Satz äußert, der sich ideal in das Sportkonzept der Zeitschrift fügt: „Beim Tennis hängt sehr viel vom Ästhetischen ab“ (ebd.). 
sind französische Avantgardekünstler, deren Sportleidenschaft Flechtheim und Wedderkop zu betonen nicht müde werden, mit Sportmotiven im „Querschnitt“ vertreten (Robert Delauney: „Die Läufer“, Ölgemälde, Der Querschnitt 5/1926: o.P.; Henri Rousseau: „Ballspieler“, Ölgemälde, Der Querschnitt 5/1926: o.P.; Lucien Maillol: „Fußball“, Ölgemälde, Der Querschnitt 8/1928: o.P.).

Das am intensivsten genutzte Repräsentationsmedium für den Sport im „Querschnitt“ bildet die Fotografie. Wie die meisten Texte und Reproduktionen bildender Kunst sind die Fotografien in der Regel anderen Zeitungen und Zeitschriften entnommen und lediglich neu zusammengestellt. Neben zahlreichen Bildern, die aktuelle Sportereignisse dokumentieren, gibt es dabei eine Reihe von Fotografien, die Sportler und Künstler gemeinsam zeigen (wie den Boxer Paul Samson-Körner mit Bertolt Brecht, Der Querschnitt 5/1926: o.P.) oder sie gleich in Personalunion vorstellen (wie in den Fällen von „Paula Heimann, [...] Breslauer Dichterin und Sportswoman“, Der Querschnitt 1/1923: o.P., oder von „Oscar Wilde's Neffe[n], de[m] Schriftsteller Cravan“, der ,,mit Jack Johnson in Barcelona 1916“ boxt, Der Querschnitt 3/1924: o.P.). Als auffälligstes Verfahren der Zusammenführung von Sport und Kunst im „Querschnitt“ erweist sich schließlich die Montage von Künstler- neben Sportlerfotografien. Ob dabei der Wiener Fußballer Peppi (,der Tank“) Uridil Marc Chagall gegenübersteht (Der Querschnitt 1/1924: o.P.), der Boxer Jack Dempsey neben dem Komponisten George Antheil aufscheint (Der Querschnitt 4/1924: o.P.) oder der indische Literatur-Nobelpreisträger Rabindranath Tagore und der Boxweltweister Gene Tunney miteinander konfrontiert werden (Der Querschnitt 11/1926: o.P.) - der Effekt bleibt stets derselbe: Immer gewinnt die Montage die Aufmerksamkeit der Leser zwar durch die Kontrastierung zweier scheinbar fremder Bildwelten; die Zusammenführung stellt Sportler und Künstler aber auf eine gemeinsame Ebene und veranlasst die Suche nach Analogien. Auf diese Suche machen sich auch die folgenden Überlegungen.

\section{Spannung und Geometrie: Sportler und Künstler als "Körperbildner"}

Das Kunstkonzept, das sich hinter der Eingliederung des Sports in den Bereich des Ästhetischen verbirgt, lässt sich anhand einiger exemplarischer Beiträge skizzieren. Im ersten „Querschnitt“-Heft des Jahres 1923 leitet Hermann von Wedderkop einen kurzen, nicht übertitelten Text über aktuelle Tendenzen der Bildhauerei mit einer Erfolgsmeldung über 
den seinerzeit bekanntesten deutschen Profiboxer und „Querschnitt“Liebling Hans Breitensträter ein:

„Unser Mitarbeiter Hans Breitensträter hat in einem schweren Kampf um die deutsche Meisterschaft im Schwergewicht Hans Wagner, Rheinlands Eiche, besiegt. Er ist jetzt von Guiseppe Spalla herausgefordert. Es könnte nun kommen, was aber kaum zu erwarten ist, daß ein Italiener deutscher Schwergewichtsmeister wird, so wie ein Italiener - de Fiori - bereits deutscher Bildhauermeister ist. - Paul Zucker schreibt in seinem Aufsatz,Plastik' in der ,Neuen Rundschau': ,Als letzte seien noch zwei Künstler genannt, deren Schaffen allein schon den Wert der plastischen Potenz unserer Zeit sichert. Die Vereinheitlichung geometrischer Körpervorstellung und der lebendigen Spannungsdynamik zwischen Masse und Raum gelingt restlos wohl nur einem unter den Jüngsten: Ernesto de Fiori [...]““ (Der Querschnitt 1/1923: 91, Hervorhebungen im Original).

Der thematische Sprung vom Boxen zur bildenden Kunst, der aufgrund des gleichzeitigen Wechsels der sprachlichen Register von der publizistischen Meldung zum kunstkritischen Essay eher gewagt wirkt, gelingt Wedderkop durch eine Überbrückung, die beide Sphären hybridisiert: Das Schaffen des seit 1921 in der Berliner Galerieszene etablierten Bildhauers Ernesto de Fiori wird zum sportlichen Wettkampf und dieser selbst zum „deutsche[n] Bildhauermeister“ erklärt, womit er auf eine Stufe mit dem ,deutsche[n] Schwergewichtsmeister“ Breitensträter rückt. Mutet diese Zusammenführung zunächst ungewöhnlich an, so erscheint sie im Kontext von Paul Zuckers Essay doch plausibel. Denn Zucker führt zwei ästhetische Kategorien ein, die als Maßstab für zeitgemäße Bildhauerei (für den ,Wert der plastischen Potenz unserer Zeit“) gelten sollen: mathematische Präzision und kraftvolle Bewegtheit. Beide lassen sich auf die einführende Boxmeldung beziehen: Eine Kunst, die eine ,geometrische[] Körpervorstellung“ mit einer „lebendigen Spannungsdynamik“ verbinden soll, findet in Körperbau und Dynamik des Boxers ihr unüberbietbares Vorbild. Boxer und Bildhauer erscheinen aus dieser Perspektive gleichermaßen als ,Körperbildner': Sie sind beide in der Lage, einen nach wissenschaftlichen Vorgaben exakt proportionierten Körper zu erschaffen (zum Zusammenhang von sportlichen und mechanischen Körpern in der Sportphysiologie vgl. den Beitrag von Michael Mackenzie in diesem Band); beiden gelingt es, körperlichen Bewegungen den Ausdruck von Impulsivität und Explosivität zu verleihen oder - wie Zucker etwas später präzisiert - zu zeigen, wie sich der Körper in den Raum ,schraubt und windet [...], federnd von der inneren Dynamik des Blutes“ (ebd.). Mit der Zusammenstellung von Boxmeldung und Skulpturessay installiert Wedderkop also das Sporttreiben subtil als 
Vorbild einer zeitadäquaten, an den Kriterien Präzision und Spannung ausgerichteten Kunst.

Die Forderung nach einer Orientierung der Kunst am Sport durch eine Darstellung, die beide als „spannungsgeladen“ attribuiert, kehrt im „Querschnitt“ immer wieder. Ein weiteres Beispiel bietet Alfred Flechtheims Beitrag „Gladiatoren“, in dem er einen Vergleich zwischen dem Boxen und dem Theater anstellt (Der Querschnitt 1/1925: 48f.). Der Galerist stellt dabei fest, dass es Theateraufführungen, die nicht bürgerlichen Bildungs- oder Amüsementzwecken dienten, zunehmend an $\mathrm{Zu}$ schauern mangele:

„Obwohl über jede Berliner Premiere die Spalten der Tagespresse von hinten bis vorne gefüllt sind, sind die Theater leer. Nur die Pelzmäntelorgien des Herrn Max Reinhardt in der Komödie und die Revuen haben [...] volle Häuser. Georg Kaisers ,Jüdische Witwe“ mußte abgesetzt werden“ (ebd.: 48).

Dem hält Flechtheim ein gesteigertes Publikumsinteresse für den Sport, insbesondere das Boxen entgegen: „Zum Kampf Hans Breitensträters mit Pablo Uzcudun waren acht Tage vorher die 15000 Plätze des Sportpalastes restlos ausverkauft" (ebd.). In ähnlicher Weise wie Bertolt Brecht, der bis Ende der zwanziger Jahre immer wieder das Theater durch eine Ausrichtung am Sport zu reformieren strebt (vgl. Sicks 2004), führt Flechtheim die von ihm erkannte Tendenz nicht etwa in kulturpessimistischer Perspektive auf einen Verfall des öffentlichen Niveaus zurück, sondern auf die steigende ästhetische Qualität des Boxens. Diese Position stützt der Galerist durch zwei Argumente. Zunächst führt er an, dass ein Boxkampf authentischer als eine Theateraufführung sei: „Was sich da in dem Ring [...] abspielt, in einer halben Stunde, ist wirkliches Drama, ist keinem Theater vergleichbar" (Der Querschnitt 1/1925: 49). Die Unterstellung einer mythischen Ursprünglichkeit - von Breitensträter heißt es auch, ,[e]r kämpfte wie Hektor nach dem Abschied von Andromache“ (ebd.) -, eines unmittelbaren Sitzes im Leben, mag auf der Annahme gründen, dass die Performativität des Sports keiner Logik der Repräsentation folge und ihm damit eine unmittelbarere Ausdrucksqualität als dem Theater innewohne. Hierin mag zugleich ein wesentlicher Grund für die Sport- und Körperbegeisterung weiterer Kreise der Zwischenkriegsavantgarde bestehen: Als Konsequenz aus den sprachskeptischen Positionen der Jahrhundertwende, für die die Einsicht in die Vermitteltheit des sprachlichen Weltzugriffs eine fundamentale metaphysische Krise auslöst (paradigmatisch sei an Hoffmannsthals Chandos-Brief erinnert), bedeutet der Rückgriff auf den Körper den Einsatz einer Ausdrucksform, die vermeintlich ohne Unterstützung eines 
semiotischen Systems emotional-psychische Befindlichkeiten artikulieren kann. Dass auch somatische Kommunikation medialen bzw. zeichenhaften Bedingungen unterworfen ist, geht in der Authentizitätseuphorie dieser Körperbegeisterung in der Regel unter.

Das zweite Argument, das Flechtheim anführt, um das Boxen als bessere Kunst zu kennzeichnen, bringt den Körper und die Spannung ins Spiel: „Boxen ist Energie in höchster Potenz“ (ebd.), erklärt Flechtheim und schließt an: „[D]ie Spannung, die in dem Ring herrscht, überträgt sich auch auf das Publikum, überträgt sich auf Max Slevogt, ebenso wie auf den Droschkenkutscher“ (ebd.). Löst man dieses Bild auf, dann steht für den sportbegeisterten Galeristen die Dynamik der sportlichen Bewegung, die Intensität der körperlichen Interaktion im Mittelpunkt seiner Boxleidenschaft. Indes begründet sich die Bewunderung der „Spannung“ aus einer besonderen Pointe: Denn erst dass die körperliche Dynamik sich als (kreative) Antriebskraft an die Zuschauer, an die Künstler (nicht zufällig nennt Flechtheim den Graphiker Slevogt, im Weiteren auch die Schauspielerin Tilla Durieux) weitervermittelt, macht ihre eigentliche Qualität aus. Die Spannung des Sports mobilisiert das Publikum, und diese Antriebsdynamik, dieses Einbeziehen der Zuschauer in das sportliche Spektakel (das auch als Form der Zusammenführung von Kunst und Leben gesehen werden kann, der klassischen Forderung europäischer Avantgardebewegungen; vgl. Asholt/Fähnders 1999: 15) wertet Flechtheim als zentrales Kennzeichen des Ästhetischen: „Aber daß dieser Kampf, in dem Kraft, Geist und Erfahrung vereint siegten, eine künstlerische Angelegenheit war, künstlerischer als alle Berliner Theateraufführungen, ist allen denen bewußt geworden, die das große Glück hatten, diesem unerhörten Schauspiel beizuwohnen. Ich beglückwünsche Breitensträter zu diesem Kampf. Der Versuch war ein kokoschkaesker“ (Der Querschnitt 1/1925: 49).

Das Verfahren der semantischen Kontamination von Sport und Kunst in diesem Artikel ist für den „Querschnitt“ insgesamt exemplarisch: Dem trainierten Körper des Sportlers und seiner Bewegung werden beständig Merkmale abgelesen, die - in den Diskurs des Ästhetischen übersetzt - ins Zentrum kunstprogrammatischer Ansätze geraten. Sportliche Körper und sportliche Körperbewegungen werden so zu Proto-Kunstwerken deklariert. Der Körper wird zum Vorbild, an dem sich die mit unterschiedlichen Medien arbeitenden Kunstdisziplinen Skulptur, Malerei, Fotografie, Theater und Literatur beim Erwerb neuer Ausdrucksformen orientieren sollen. „Die Elemente der neuen Ästhetik sind dieselben wie beim Sport", schreibt Wedderkop 1926 (Der Querschnitt 7/1926: 497) und bringt damit sein Hauptanliegen auf den Punkt: die Kunst durch eine Orientierung an der modernsten Form körperlicher Er- 
tüchtigung zu reformieren und ihre Kennzeichen - Genauigkeit, Authentizität, Spannung - zu Maßstäben ästhetischer Qualität zu deklarieren.

\section{"Sachlicher Auftritt": Amerikanismus in Sport und Kunst}

Welche Relevanz der „Spannung“ als ästhetischer Kategorie auf der Ebene des gedruckten Wortes oder Bildes innewohnen kann, verdeutlicht der „Querschnitt“" selbst. So besteht das charakteristische Profil der Zeitschrift in der weitgehend unvermittelten Montage der Texte und Bilder neben- und ineinander, in der fast nie expliziten, in der Regel aber offenkundigen Bezugnahme einzelner Beiträge aufeinander. Texte und Bilder werden in den Kontext anderer Texte und Bilder eingegliedert, die ihren Sinn entscheidend mitbestimmen. Gerade die semantisch unterschiedlich stark „aufgeladenen“ Relationen zwischen verschiedenen „Polen“ der Zeitschrift, die sich durchaus mit der physikalischen Metapher der „Spannungen“ beschreiben lassen, bilden den Kern der „Querschnitt"-Ästhetik. ${ }^{4}$ Medientheoretisch gesprochen entsteht Bedeutung damit durch intra- und intermediale Differenz; die „Um-, Ein- und ÜberSchreibungen“, die im Rahmen solcher Bezugnahmen entstehen, hat Ludwig Jäger als „Transkriptionen“ bezeichnet (Jäger 2004: 71, Hervorhebungen im Original). ${ }^{5}$ Die transkriptive Logik des „Querschnitts“ ist nicht zuletzt für die folgende Analyse eines erneut dem Boxstar Breitensträter gewidmeten Artikels aus der Feder Hermann von Wedderkops von Bedeutung.

Der Beitrag erscheint in der Septemberausgabe des Jahrgangs 1921 und ist mit fünf Fotografien illustriert. Drei dieser Fotos sind kleinformatig und in den Text eingelassen, zwei sind größer und stehen einander auf einer eigenen Doppelseite gegenüber (Abb. 1). Das erste kleine Foto (Der Querschnitt 4-5/1921: 137) zeigt den Boxer in Siegerpose, nur mit Sporthose, Boxhandschuhen und Schuhen bekleidet, aber mit einem riesigen Kranz, einem Mantel und einem Handtuch behängt. Solchermaßen

4 In den fünfziger Jahren knüpft die Kulturzeitschrift „Magnum“ im Rahmen eines auf den ersten Blick paradox wirkenden konservativ-modernistischen Programms an diese Bild-Text-Struktur des „Querschnitts“ an und nutzt ebenfalls die „Spannung“ zwischen einzelnen (zumeist fotografischen) Beiträgen als zentrales Gestaltungsprinzip (vgl. Starl 2004: 130-136).

5 Aufgrund der changierenden Bezugnahmen unterschiedlicher medialer Systeme aufeinander ließe sich das transkriptive Verfahren des "Querschnitts“ weiterführend als ,oszillierende[r] Typus wechselseitigen Kommentierens“ beschreiben (Jäger 2004: 74, Hervorhebung im Original; zur Transkriptivität als der Grundlage kultureller Semantik im Allgemeinen vgl. Jäger 2002). 




Abb. 1: Bilddoppelseite aus dem „Querschnitt“, Heft 4-5/1921.

mit dem vollständigen ikonographischen Inventar des Boxens ausgerüstet, steht das Bild in scharfem Kontrast zu den beiden anderen kleinformatigen Fotos, die am Ende in den Text eingelassen sind. Hier ist der Boxer mit jeweils anderen Personen aufgenommen, fällt aber durch seine alltägliche Kleidung (Trenchcoat, Hut) nicht als Sportler auf. Auf den ersten Blick müsste man ihn aufgrund seiner Begleitung vielmehr (einmal mehr) einer anderen Kulturpraxis zuordnen: der bildenden Kunst. So ist Breitensträter zunächst mit der Bildhauerin Renée Sintenis und den Malerinnen Gertrud Sauermann und Susi von Zimmermann zu sehen (ebd.: 140); das andere Mal flaniert er an der Seite Ernesto de Fioris durch einen Park (ebd.: 141). Bezieht man die Bilder aufeinander und beachtet dabei ihre Anordnung, dann lässt sich eine narrative Struktur erkennen: Die Fotos erzählen en miniature von der Verwandlung Breitensträters vom Boxer zum Künstler, vom mit allen Attributen der Zunft ausgestatteten Sportchampion zum intellektuellen Mitglied der Berliner Avantgarde; allgemeiner gesagt: Die Bilder illustrieren die Integration des Sports in die Kunstkreise um den Galeristen Alfred Flechtheim.

Diese Narration wird in den beiden großen Bilder auf der in die Mitte des Artikels eingelassenen Bilddoppelseite wiederholt; die Montage erscheint durch ihre zentrale Platzierung als Kern der Verwandlungssze- 
nerie. Auf der rechten Seite ist Hans Breitensträter in konzentriertangespannter Kämpferpose zu sehen, diesmal nur mit einer kurzen weißen Hose bekleidet. Auffällig ist das im „Querschnitt“ ungewöhnliche Oval-Format des Fotos. Auf der linken Seite wird der bereits über 70jährige Auguste Renoir gezeigt, der im Rollstuhl an einem Gemälde der Schauspielerin Tilla Durieux arbeitet. Im Hintergrund lässt sich Renoirs Atelier mit einigen weiteren Gemälden ausmachen.

Eine eingehende Lektüre offenbart ein komplexes Beziehungsgefüge zwischen beiden Fotografien. Zunächst verweisen die Bildunterschriften auf eine Gemeinsamkeit der Abbildungen: „AUGUSTE RENOIR malt Tilla Durieux im Frühjahr 1914“, heißt es unter der einen; „HANS BREITENSTRÄTER (der deutsche Schwergewichtsmeister schreibt für Rud. Großmanns Boxermappe das Vorwort)“, lautet der Untertitel des anderen Bildes. Breitensträter und Renoir werden hier auf eine Ebene geführt, indem beider Namen groß geschrieben und beiden künstlerische Tätigkeiten zugeordnet werden: Malen, Schreiben. Bei Renoir hätte es dieser Explikation kaum gebraucht, seine Arbeit am weiblichen Porträt weist ihn für jeden Betrachter zweifelsfrei als Künstler aus. Breitensträter indes ist (kaum überraschend) nicht bei der ihm zugeschriebenen Schreibtätigkeit zu sehen; boxend trainiert er seinen Körper. Andere Kontraste zwischen den Fotografien fallen ins Auge. Der gebrechlichen Konstitution Renoirs, der zum Zeitpunkt der Gründung des „Querschnitts“ bereits seit zwei Jahren tot ist, steht der durchtrainierte Körper des Boxers gegenüber; dem Alter des Malers die Jugend Breitensträters. Ist Breitensträter der Proponent einer neuen Form des Kunstschaffens?

Allerdings lässt sich das Foto des Boxers nicht nur auf die Abbildung Renoirs beziehen, sondern ebenso auf das bildinterne Gemälde der Tilla Durieux. Insbesondere die ovale Rahmung, die das Foto wie ein Amulettbild wirken lässt und seine Porträthaftigkeit in den Vordergrund stellt, schafft eine Analogie zum Abbild der exzentrischen Schauspielerin und grande dame der Berliner Kunstszene. Wie Tilla Durieux verdient Breitensträter zudem sein Geld durch öffentliche Auftritte, wie die Schauspielerin zeigt er seine Kunst vor großem Publikum. In der Sichtweise der hier versuchten Auslegung der Fotofolge verwandelt sich Breitensträter darum nicht nur in einen Künstler, sondern zugleich in sein eigenes Kunstwerk. Die Bilder analogisieren Sport und Kunst in doppelter Weise: der Sportler ist Schöpfer und Werk zugleich (zu einer ähnlichen Doppelfigur vgl. den Beitrag von Maren Möhring in diesem Band).

Der Text, der die Bilder umgibt, unterstützt diese Lesart. Gleichzeitig bringt er einige Schlagworte ins Spiel, die noch deutlicher die ästhetische Programmatik erkennen lassen, die sich hinter der Sport-Kunst- 
Kontraktion verbirgt. Wedderkop beschreibt hier seine erste Begegnung mit dem Boxer, den er in Begleitung Flechtheims und des Malers Rudolf Grossmann in seiner Berliner Wohnung aufsucht. Er schildert Breitensträter dabei einerseits als längst eingeführtes Mitglied der Künstlerkreise um den Galeristen Flechtheim, ${ }^{6}$ andererseits erweist er sich in der Beschreibung des Boxerkörpers vollends als Kunstkritiker: Im Duktus einer Kunstwerkswürdigung berichtet Wedderkop vom ersten „Auftritt“ des Sportlers und stellt fest, dass Breitensträter weder eine klassischantike Figur habe noch sich anderen aus der Kunst bekannten Schönheitsidealen füge:

„Er ist auch nicht griechisch. Nicht die Spur. Kein Grieche aus Sachsen. Alles Erinnern unser durch klassisches Klischee vergifteten Gehirns muss zerstört werden. Festgehaltene Bilder müssen Platz machen, damit ein neues Bild geprägt wird. [...] Ausserdem: von Myron hat er nichts, von dessen scharfer Schwere, noch von dem öligen Praxiteles. Auch keine Mischung. Er stammt aus Magdeburg. Eher können wir ihn auf einen amerikanischen Grundbegriff bringen" (ebd.: 140).

„Amerikanisch“ wird hier zu Wedderkops Metapher eines nachklassischen, zeitgemäßen Körperideals. Diesem „Grundbegriff“ werden andere Attribute zur Beschreibung des Körpers untergeordnet: „mechanisiert[]“ (ebd.: 138), ,sachlich“ (ebd.: 139 und 140) und „elastisch“ (ebd.: 138 und 139). Breitensträters Bewegungen seien sich ihrer selbst „noch unbewusst“ (ebd.: 141), bewegten sich aber ,auf der Kippe zwischen weich und hart, gerade im günstigen Moment, wo aus unbewusster Genussbewegung harter, berechneter Zweck wird" (ebd.: 139). Schließlich sei Breitensträters Auftreten durch „Gleichmut“ und „Kühle“ (ebd.: 140) gekennzeichnet. Diese Zuschreibungen sind in doppelter Hinsicht aufschlussreich: Sie führen einerseits das Sportverständnis Wedderkops vor Augen; andererseits ermöglichen sie, dieses Sportverständnis in Relation zu einem spezifischen zeitgenössischen Kunstdiskurs zu setzen und so die Hintergründe für die Sport-Kunst-Parallelen im „Querschnitt“ zu verstehen.

Zunächst wird Breitensträter in Wedderkops Augen zum Prototypen einer „kalten persona“, die Helmut Lethen als zentralen Protagonisten

6 Breitensträters Integration in die „Querschnitt“-Gesellschaft scheint in der Tat weit gegangen zu sein, was sich nicht zuletzt daran erweist, dass er verschiedene Male selbst Beiträge in der Zeitschrift publizieren darf. Dabei geht es nicht immer nur um Boxkämpfe. Noch im Jahr 1932 etwa erörtert Breitensträter die anscheinend heikle Frage „Soll ein Sportsmann heiraten?“ (Der Querschnitt 6/1932: 394f.) mit einem, ,glatten Ja. Er soll heiraten, aber nur eine vernünftige Frau“ (ebd.: 394). 
des literarischen Diskurses der zwanziger Jahre dargestellt hat (vgl. Lethen 1994). Die „kalte persona“ inkorporiert in radikaler Weise die „Verhaltenslehren der Kälte“, die nach Lethen in „Augenblicken sozialer Desintegration, in denen die Gehäuse der Tradition zerfallen und Moral an Überzeugungskraft einbüßt“, zum allgemeinen Maßstab werden (ebd.: 7). Diese Verhaltenslehren schreiben ein nüchternes, unemotionales, das psychische Befinden verbergendes Auftreten vor, um klare Grenzen zwischen dem Eigenen und dem Fremden zu setzen sowie Beschäftigungen mit einem eventuell schuldhaft verstrickten Selbst den Riegel vorzuschieben. Indes scheint Lethen zufolge das sachliche Verhalten dort an seine Grenzen zu stoßen, wo sich der Mensch nicht unbeschränkt als formbar erweist - am Körper: „Es kennzeichnet die Literatur am Ende der Republik, daß in ihr die Handlungsphantasien nicht auf moralischen Einspruch treffen, sondern auf den Widerstand der Körperwelt"“ (ebd.: 13). In Breitensträter ist dieser Widerstand aufgegeben: Sein Körper ist vollständig nach den maschinenhaften Vorgaben der Kältelehren aufgebaut, fügt sich gänzlich in die Doktrin der Sachlichkeit ein. Der Boxer ist die Vollendung der „kalten persona“. ?

Von Wedderkops Beschreibung des Boxers Breitensträter ausgehend, lässt sich damit die Spur zu einem zentralen kunsttheoretischen Diskurs verfolgen, der im Hintergrund der Sportbegeisterung des „Querschnitts“ steht. Denn in Wedderkops Rede über den Sportler werden exakt jene Begrifflichkeiten wiederholt, die die poetologischen Schriften neusachlicher Ästhetik auszeichnen. Die Neue Sachlichkeit als eine sich zu Beginn der 1920er Jahre im Anschluss an frühere Überlegungen Döblins und Loos' formierende Kunsttheorie hat zwar nie eine Institutionalisierung (etwa durch die Bildung von „Schulen“) erfahren; dennoch erweisen sich die unter ihrem Signum firmierenden Konzepte als weitgehend homogen (vgl. Becker 2000: 4-16; Hermand 1978). Insbesondere bildende Kunst und Literatur sind die Felder, auf denen die antiexpressionistischen und antiästhetizistischen Debatten um den dokumentarischen Stil der Neuen Sachlichkeit bis 1933 ausgetragen werden. Bei einer prinzipiellen Bejahung der industrialisierten und urbanisierten Moderne

7 Diese Verbindung lässt sich erweitern, wenn man andere Artikel des ,Querschnitts" berücksichtigt: Sport bildet hier immer wieder auch eine Chiffre für industrialisierte Modernität. Aufgrund der in ihm zur Geltung kommenden genormten und disziplinierten Körper(bewegungen) wird Sport als maschinenhaft präzise wahrgenommen, seine reine Leistungsbezogenheit mit Sachlichkeit und Nüchternheit assoziiert und der Wettkampfcharakter mit kapitalistischer Konkurrenz gleichgesetzt. Die Rede über Sport erscheint so als, wie Frank Becker dies nennt, „Interdiskurs“, der die abstrakten Anforderungen des modernen Geschäftslebens in eine alltägliche Sprache übersetzt (vgl. Becker 1993: 336-351). 
setzt der neusachliche Diskurs der Kunst zum Ziel, eine präzise und nüchterne „Abbildung“ der komplexen modernen „Wirklichkeit“ (die Strömung bezeichnet sich selbst auch als „Neuen Naturalismus“, vgl. Becker 2000: 108-116) bei Ausklammerung jedweder psychologischen Charakterschilderung und wertenden Tendenz zu leisten. Am Ende soll das Bild einer „dynamischen“, durch verschiedenste „Spannungen“ gekennzeichneten Gegenwart stehen, das deren Anatomie in so evidenter Weise offenbart, dass sich Bewertungen in der Rezeption von selbst ergeben (vgl. ebd.: 187-205). In Wedderkops Beschreibung des Sportlers überlagert sich somit die Kennzeichnung Breitensträters als „,kalte persona" mit der Einschätzung seines Körpers als eines idealen neusachlichen Kunstwerks. Breitensträter ist wie die Kunst der Neuen Sachlichkeit durch Nüchternheit (,sachlich“), Antisentimentalität (,kühl“), Dis$\operatorname{tanz}($,Gleichmut") und Präzision (,mechanisiert“) gekennzeichnet (vgl. Becker 2000: 97-258; Lethen 1991; Petersen 1982).

Verallgemeinert man diese Erkenntnis, dann lässt sich „Der Querschnitt" als ein wesentliches Organ neusachlicher Theoriebildung in den zwanziger Jahren verstehen. Die Koppelung von Kunst und Sport erscheint dann als selbständiger Beitrag zur Veranschaulichung einer sachlichen, objektiven, präzisen und spannungsgeladenen Kunst. Sport, als Symbol und Vorbild einer reformierten Kunst gezeichnet, garantiert dieser Kunst zugleich ihre Publikumsverbundenheit und Alltäglichkeit und öffnet das neusachliche Theorieprojekt des „Querschnitts“ so in Richtung anderer Projekte der europäischen Avantgarde.

\section{Literaturverzeichnis}

Asholt, Wolfgang/Fähnders, Walter (2000): Einleitung. In: Dies. (Hg.):

Der Blick vom Wolkenkratzer. Avantgarde - Avantgardekritik Avantgardeforschung, Amsterdam u.a.: Rodopi, S. 9-27.

Becker, Frank (1993): Amerikanismus in Weimar. Sportsymbole und politische Kultur 1918-1933, Wiesbaden: Deutscher Universitätsverlag.

Becker, Sabina (2000): Neue Sachlichkeit. Band 1: Die Ästhetik der neusachlichen Literatur (1920-1933), Köln u.a.: Böhlau.

Berg, Günter (1997): „Die Männer boxen im Salatgarten“. Bertolt Brecht und der Faustkampf. The Brecht Yearbook 18: The Other Brecht II, S. 17-39.

Berg, Günter (1998): „Nachwort“. In: Bertolt Brecht, Der Kinnhaken und andere Box- und Sportgeschichten, Frankfurt a.M.: Suhrkamp, S. 131-151. 
Deininger, Bettina/Felder, Ulrike (1998): „,Der Stoff liegt auf der Straße. 'Der Querschnitt“. In: Patrick Rössler (Hg.), Moderne Illustrierte. Illustrierte Moderne. Zeitschriftenkonzepte im 20. Jahrhundert, Stuttgart: Württembergische Landesbibliothek, S. 26-37.

Der Querschnitt. Berlin 1921-1936.

Hermand, Jost (1978): „Einheit in der Vielheit? Zur Geschichte des Begriffs ,Neue Sachlichkeit““. In: Ders., Stile, Ismen, Etikette. Zur Periodisierung der modernen Kunst, Wiesbaden, S. 80-93.

Jäger, Ludwig (2002): „Transkriptivität. Zur medialen Logik der kulturellen Semantik“. In: Ders./Georg Stanitzek (Hg.), Transkribieren Medien/Lektüre, München: Fink, S. 19-41.

Jäger, Ludwig (2004): „Die Verfahren der Medien: Transkribieren - Adressieren - Lokalisieren." In: Jürgen Fohrmann/Erhard Schüttpelz (Hg.), Die Kommunikation der Medien, Tübingen: Niemeyer, S. 6979.

Lethen, Helmut (1991): „Der Jargon der Neuen Sachlichkeit“. Germanica 9 (Themenband „Die ,Neue Sachlichkeit‘. Lebensgefühl oder Markenzeichen?“), S. 11-36.

Lethen, Helmut (1994): Verhaltenslehren der Kälte. Lebensversuche zwischen den Kriegen, Frankfurt a.M.: Suhrkamp.

Petersen, Klaus (1982): „,Neue Sachlichkeit“: Stilbegriff, Epochenbezeichnung oder Gruppenphänomen“. DVjs 56 (Heft 3), S. 463-477.

Sicks, Kai Marcel (2004): „Sollen Dichter boxen? Brechts Ästhetik und der Sport". Hofmannsthal-Jahrbuch 13, S. 363-404.

Starl, Tim (2004): ,,Der ewige Mensch“. Karl Pawek und die ,Weltausstellungen der Photographie“"“ In: Jean Back/Viktoria SchmidtLinsenhoff (Hg.), The Family of Man 1955-2001 - Humanismus und Postmoderne. Eine Revision von Edward Steichens Fotoausstellung, Marburg: Jonas. 